\title{
Investigation of Preferred Orientations in Planar Polycrystals
}

\author{
M. R. Tonks, ${ }^{1,2}$ A. J. Beaudoin,${ }^{2}$ F. Schilder ${ }^{3}$ and D. A. Tortorelli ${ }^{2}$ \\ ${ }^{1}$ T-3, Los Alamos National Laboratory, Los Alamos, NM, 87545, USA \\ ${ }^{2}$ Department of Mechanical Sciences and Engineering, \\ University of Illinois at Urbana-Champaign, Urbana, IL 61801, USA \\ ${ }^{3}$ Department of Mathematics, University of Surrey, Guildford, GU2 $7 X H$ UK, \\ supported by EPSRC grants GR/R72020/01 and EP/D063906/1
}

(Dated: February 11, 2008)

\begin{abstract}
More accurate manufacturing process models come from better understanding of texture evolution and preferred orientations. We investigate the texture evolution in the simplified physical framework of a planar polycrystal with two slip systems used by Prantil et al. (1993, J. Mech. Phys. Solids, 41(8), 1357-1382). In the planar polycrystal, the crystal orientations behave in a manner similar to that of a system of coupled oscillators represented by the Kuramoto model. The crystal plasticity finite element method (CPFEM) and the stochastic Taylor model (STM), a stochastic method for mean-field polycrystal plasticity, predict the development of a steady-state texture not shown when employing the Taylor hypothesis. From this analysis, the STM appears to be a useful homogenization method when using representative standard deviations.
\end{abstract}

Keywords: Polycrystal Plasticity, Planar Polycrystal, Stochastic Taylor Model, Kuramoto Model 


\section{INTRODUCTION}

Crystallographic textures, which evolve during manufacturing processes, are central to the anisotropic response of the processed material. These textures do not evolve randomly, but rather towards certain preferred orientations determined by the crystal structure, loading, and other factors. As such, understanding the development and nature of preferred orientation is of critical importance when developing meaningful models of manufacturing processes. This is a complicated task because the driving forces behind the evolution cover multiple length scales ${ }^{1}$. To bridge the length-scales, it is often convenient to consider the polycrystalline material as an aggregate of anisotropic meso-scale crystals.

Mean-field polycrystal plasticity models describe the aggregate response to an applied deformation by predicting the response of each crystal with a meso-scale model. These mesoscale fields are subsequently homogenized to obtain the macro-scale response, including the stress in the aggregate and the evolving texture. However, a method is needed to relate the macro-scale deformation to the meso-scale deformations experienced by the crystals. Several such methods appear in the literature, the most common being the fully constrained model (FCM), based on the hypothesis in Taylor ${ }^{2}$, which asserts that the crystal deformations are equal to the macroscopic deformation. Many applications of this hypothesis validate its use $^{3,4}$, however, it is not consistent with the physical behavior of polycrystalline materials. Several alternative methods have been proposed ranging from relaxed constraint methods in which only selected components of the macro-scale and crystal deformation rate and stress are equated $^{5-7}$ to self-consistent models ${ }^{8}$ in which a grain is introduced in a homogeneous effective medium which provides the average response of all grains in the aggregate. Of course, the increased accuracy of these models comes at the expense of computational complexity.

Prantil et al. ${ }^{9}$ apply mean-field polycrystal plasticity to a simplified planar polycrystal in order to investigate texture evolution and preferred orientations. By using 2 slip systems to describe the plastic deformation in the planar single crystals, they develop an analytical expression defining the reorientation of a crystal throughout an imposed deformation. Using the FCM to relate deformations between the crystals and the macro-scale, they find that the texture of a deformed planar polycrystal either approaches a single, preferred orientation or periodically oscillates, depending on the magnitude of the spin in the applied deformation. Kumar and Dawson ${ }^{10}$ conduct a similar investigation on planar polycrystals, but look at 
single crystals with two to four slip systems. As with Prantil et al. ${ }^{9}$, they utilize the FCM. They show that as the number of slip systems increase, the number of possible texture evolution behaviors also increase, but each behavior is still either an evolution towards a single orientation or a periodic evolution. Kumar ${ }^{11}$ shows that $3 \mathrm{D}$ cubic polycrystals modeled with the FCM loaded under pure shear experience five stable orientations at which the texture will continue to increase in strength, but when a spin is introduced there are no stable orientations and the texture may oscillate in strength. Therefore, while crystals tend to reorient toward preferred orientations, a significant applied spin can induce periodic reorientation. In general, the FCM is considered to predict exaggerated texture evolution, since it over-constrains the crystals.

In this work, we investigate the development of preferred orientations using the FCM, as well as two additional methods that relax the strict equality between the macro- and meso-scale deformation rates. Our objective is to gain understanding into the dynamical nature of a preferred orientation, as well as the evolution towards this orientation. As more complex systems show similar trends to simpler idealizations, we investigate the texture evolution behavior in an idealized planar polycrystal with two slip systems. We apply the crystal plasticity finite element method (CPFEM), where each crystal in the aggregate is represented by one or more finite elements ${ }^{12-16}$, as well as a stochastic mean-field model ${ }^{17,18}$ to the deformation of the planar aggregate. We begin in sections II and III, presenting the single crystal model for the planar polycrystal. In section IV we compare the texture trajectory to that of a system of coupled oscillators represented by the Kuramoto model, in turn revealing a link between the development of preferred orientations and the notion of synchronization. We compare the FCM texture evolution to that predicted from the CPFEM using an idealized microstructure in Section V. In sections VI and VII, We develop a stochastic method and compare the predicted behavior to the CPFEM results. Both the CPFEM and the stochastic model show the evolution toward a stable texture in the presence of macro-scale spin. In both cases, this "stable" texture exhibits a dynamic character, in which component crystals continue crystallographic reorientation but the overall distribution does not change. Section VIII investigates the long-term behavior of the texture evolution and discusses the applicability of the results to planar polycrystals with more slip systems, as well as to $3 \mathrm{D}$ polycrystals. 


\section{GENERAL ROTATION EVOLUTION EQUATION}

Following Kok et al. ${ }^{19}$ we derive an expression for the evolution of the crystal lattice rotation of a crystal $c$ as a function of the current state variables (crystal lattice rotation and "flow stress") and the current crystal velocity gradient $\mathbf{L}^{c}$. To simplify our analysis we neglect elastic effects.

A material point in a crystal $c$ identified by position vector $\mathrm{x}^{c}$ in the reference configuration is mapped to the location in the deformed configuration at time $t$ identified by the position vector $\mathrm{x}_{t}^{c}$ through the motion $\mathbf{f}^{c}$, i.e. $\mathrm{x}_{t}^{c}=\mathbf{f}^{c}\left(\mathrm{x}^{c} ; t\right)$. We define the deformation gradient as the spatial derivative, i.e. $\mathbf{F}^{c}=\nabla \mathbf{f}^{c}$ with $\mathbf{F} \in \operatorname{Lin}^{+}$where $\operatorname{Lin}^{+}$is the set of tensors with positive determinant. As per usual, we require $\operatorname{det} \mathbf{F}^{c}>0$ so that the mapping $\mathbf{f}^{c}$ at a given time $t$ is everywhere invertible.

The material point velocity can be defined with respect to the reference configuration, giving the material velocity, or with respect to the deformed configuration, giving the spatial

velocity, i.e. $\dot{\mathbf{f}}^{c}\left(\mathrm{x}^{c} ; t\right)=\mathrm{v}^{c}(\overbrace{\mathrm{x}_{t}^{c}}^{\mathbf{f}^{c}\left(\mathrm{x}^{c} ; t\right)} ; t)$. Differentiating this equality with respect to the reference position $\mathrm{x}^{c}$ and rearranging gives the expression for the spatial crystal velocity gradient

$$
\mathbf{L}^{c}=\nabla \mathrm{v}^{c}=\dot{\mathbf{F}}^{c} \mathbf{F}^{c-1}
$$

The crystal deformation gradient is assumed to be the result of a plastic deformation followed by a rigid rotation, i.e.

$$
\mathbf{F}^{c}=\mathbf{R}^{c} \mathbf{F}_{p}^{c} \quad \text { with } \mathbf{R}^{c} \in \operatorname{Rot} \text { and } \operatorname{det} \mathbf{F}_{p}^{c}=1
$$

where Rot is the set of all rotations. $\mathbf{F}_{p}^{c}$ represents the plastic isochoric deformation gradient due to motion along slip planes in the crystal lattice and $\mathbf{R}^{c}$ represents the crystal lattice rotation. Substituting Eq. (2) into Eq. (1), we obtain

$$
\mathbf{L}^{c}=\dot{\mathbf{R}}^{c} \mathbf{R}^{c T}+\mathbf{R}^{c} \mathbf{L}_{p}^{c} \mathbf{R}^{c T}
$$

where $\mathbf{L}_{p}^{c} \equiv \dot{\mathbf{F}}_{p}^{c} \mathbf{F}_{p}^{c-1}$ is the plastic velocity gradient. The symmetric $\mathbf{D}^{c}$ and skew $\mathbf{W}^{c}$ components of $\mathbf{L}^{c}$ are

$$
\begin{aligned}
\mathbf{D}^{c} & =\mathbf{R}^{c} \mathbf{D}_{p}^{c} \mathbf{R}^{c T} \\
\mathbf{W}^{c} & =\dot{\mathbf{R}}^{c} \mathbf{R}^{c T}+\mathbf{R}^{c} \mathbf{W}_{p}^{c} \mathbf{R}^{c T} .
\end{aligned}
$$




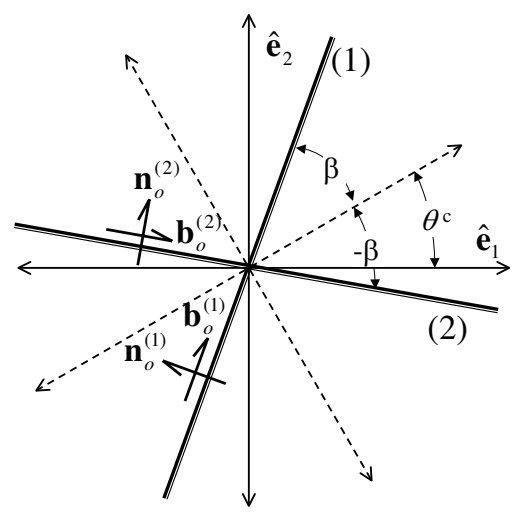

FIG. 1: Single planar crystal structure.

where $\mathbf{D}_{p}^{c}$ and $\mathbf{W}_{p}^{c}$ are the symmetric and skew components of $\mathbf{L}_{p}^{c}$, respectively. The evolution equation of the lattice rotation is obtained by solving Eq. (5) for $\dot{\mathbf{R}}^{c}$ giving

$$
\dot{\mathbf{R}}^{c}=\mathbf{W}^{c} \mathbf{R}^{c}-\mathbf{R}^{c} \mathbf{W}_{p}^{c} .
$$

For a single crystal, the plastic velocity gradient is assumed to be given by

$$
\mathbf{L}_{p}^{c}=\sum_{s=1}^{M} \dot{\gamma}^{s} \mathbf{S}_{o}^{s}
$$

where, for each of the $M$ slip systems $s, \dot{\gamma}^{s}$ is the shear rate, $\mathbf{S}_{o}^{s}=\mathbf{b}_{o}^{s} \otimes \mathbf{n}_{o}^{s}$ is the Schmidt tensor, and $\mathbf{b}_{o}^{s}$ and $\mathbf{n}_{o}^{s}$ are unit vectors for the unrotated, i.e undeformed, crystal along the slip direction and normal to the slip plane, respectively. Expressions for $\mathbf{D}_{p}^{c}$ and $\mathbf{W}_{p}^{c}$ are obtained from Eq. (7) as

$$
\begin{aligned}
\mathbf{D}_{p}^{c} & =\sum_{s=1}^{M} \dot{\gamma}^{s} \mathbf{m}_{0}^{s} \\
\mathbf{W}_{p}^{c} & =\sum_{s=1}^{M} \dot{\gamma}^{s} \mathbf{q}_{o}^{s}
\end{aligned}
$$

with $\mathbf{m}_{0}^{s}$ and $\mathbf{q}_{o}^{s}$ being the symmetric and skew components of the Schmidt tensor, respectively.

\section{PLANAR AGGREGATE MODEL}

From Eq. (6), we derive an equation for the planar crystal aggregate motion as shown in Prantil et al. ${ }^{9}$. The crystals in the aggregate have $M=2$ slip systems such that the 
partition of shearing among the slip systems is kinematically determined. The slip systems are portrayed in their deformed configuration in Fig. 1 where the Cartesian coordinate system is defined by the orthonormal basis vectors $\left(\hat{\mathbf{e}}_{1}, \hat{\mathbf{e}}_{2}, \hat{\mathbf{e}}_{3}\right)$ with $\hat{\mathbf{e}}_{3}$ normal to the page. Each crystal $c$ in the aggregate experiences a rotation of the angle $\theta^{c}$ about $\hat{\mathbf{e}}_{3}$, i.e.

$$
\begin{aligned}
\mathbf{R}^{c}=\mathbf{R}\left(\theta^{c}\right)= & \hat{\mathbf{e}}_{3} \otimes \hat{\mathbf{e}}_{3}+\cos \theta^{c}\left(\hat{\mathbf{e}}_{1} \otimes \hat{\mathbf{e}}_{1}+\hat{\mathbf{e}}_{2} \otimes \hat{\mathbf{e}}_{2}\right)- \\
& \sin \theta^{c}\left(\hat{\mathbf{e}}_{1} \otimes \hat{\mathbf{e}}_{2}-\hat{\mathbf{e}}_{2} \otimes \hat{\mathbf{e}}_{1}\right) .
\end{aligned}
$$

The individual crystal deformation is due to slip over the two independent slip systems $(s=1,2)$, with

$$
\begin{aligned}
& \mathbf{b}_{o}^{(1)}=\mathbf{R}(\beta) \hat{\mathbf{e}}_{1} \\
& \mathbf{n}_{o}^{(1)}=\mathbf{R}(\beta) \hat{\mathbf{e}}_{2} \\
& \mathbf{b}_{o}^{(2)}=\mathbf{R}(-\beta) \hat{\mathbf{e}}_{1} \\
& \mathbf{n}_{o}^{(2)}=\mathbf{R}(-\beta) \hat{\mathbf{e}}_{2} .
\end{aligned}
$$

where $\beta$ is known (cf. Fig. 1).

For general isochoric planar motions, the velocity gradient can be written as a function of three independent components, the rate of stretching $\Lambda^{c}$, the rate of shearing $\Gamma^{c}$, and the $\operatorname{spin} \Omega^{c 1}$, i.e.

$$
\begin{aligned}
\mathbf{L}^{c}= & \Lambda^{c}(t)\left(\hat{\mathbf{e}}_{1} \otimes \hat{\mathbf{e}}_{1}-\hat{\mathbf{e}}_{2} \otimes \hat{\mathbf{e}}_{2}\right)+\Gamma^{c}(t)\left(\hat{\mathbf{e}}_{1} \otimes \hat{\mathbf{e}}_{2}+\hat{\mathbf{e}}_{2} \otimes \hat{\mathbf{e}}_{1}\right)+ \\
& \Omega^{c}(t)\left(\hat{\mathbf{e}}_{1} \otimes \hat{\mathbf{e}}_{2}-\hat{\mathbf{e}}_{2} \otimes \hat{\mathbf{e}}_{1}\right) \\
\mathbf{D}^{c}= & \Lambda^{c}(t)\left(\hat{\mathbf{e}}_{1} \otimes \hat{\mathbf{e}}_{1}-\hat{\mathbf{e}}_{2} \otimes \hat{\mathbf{e}}_{2}\right)+\Gamma^{c}(t)\left(\hat{\mathbf{e}}_{1} \otimes \hat{\mathbf{e}}_{2}+\hat{\mathbf{e}}_{2} \otimes \hat{\mathbf{e}}_{1}\right) \\
\mathbf{W}^{c}= & \Omega^{c}(t)\left(\hat{\mathbf{e}}_{1} \otimes \hat{\mathbf{e}}_{2}-\hat{\mathbf{e}}_{2} \otimes \hat{\mathbf{e}}_{1}\right) .
\end{aligned}
$$

To solve for the texture evolution $\dot{\theta}^{c}$, we first find $\dot{\gamma}^{s}(s=1,2)$ by substituting Eqs. (8), (10), (11), and (13) into Eq. (4) to obtain

$$
\begin{aligned}
& \dot{\gamma}^{1}=2 \csc 4 \beta\left(\Gamma^{c} \sin 2\left(\beta-\theta^{c}\right)-\Lambda^{c} \cos 2\left(\beta-\theta^{c}\right)\right) \\
& \dot{\gamma}^{2}=2 \csc 4 \beta\left(\Gamma^{c} \sin 2\left(\beta+\theta^{c}\right)+\Lambda^{c} \cos 2\left(\beta+\theta^{c}\right)\right) .
\end{aligned}
$$

\footnotetext{
${ }^{1}$ Note that these definitions are slightly different than those employed by Prantil et al. ${ }^{9}$, in which $\dot{\Lambda}^{c}$ is the rate of stretching and $\dot{\Gamma}^{c}$ is the rate of shearing.
} 
We then substitute Eqs. (9), (10), (11), (14), and (15) into Eq. (6), giving the evolution equation for the crystal orientation

$$
\dot{\theta}^{c}=-\Omega^{c}+\lambda\left(\Gamma^{c} \cos 2 \theta^{c}-\Lambda^{c} \sin 2 \theta^{c}\right)
$$

where

$$
\lambda \equiv \sec 2 \beta
$$

To achieve an expression for the current crystal orientation $\theta^{c}$ whose initial orientation is $\theta_{0}^{c}$, Eq. (16) is integrated. Assuming $\Gamma^{c}, \Lambda^{c}$, and $\Omega^{c}$ are constant, the integration gives

$$
\begin{aligned}
\theta^{c}=\tan ^{-1} & {\left[\left(-\lambda \Lambda^{c}+\mathcal{F} \tanh \left[t \mathcal{F}+\tanh ^{-1}\left[\frac { 1 } { \mathcal { F } } \left(\lambda \Lambda^{c}+\right.\right.\right.\right.\right.} \\
& \left.\left.\left.\left.\left.\left(\lambda \Gamma^{c}+\Omega^{c}\right) \tan \theta_{0}^{c}\right)\right]\right]\right)\left(\Gamma^{c} \lambda+\Omega^{c}\right)^{-1}\right],
\end{aligned}
$$

where $\mathcal{F}=\sqrt{\lambda^{2}\left(\Gamma^{c}{ }^{2}+\Lambda^{c 2}\right)-\Omega^{c} 2}$. For our subsequent analyses we find it convenient to "invert" the above to obtain an expression for

$$
\begin{aligned}
\theta_{0}^{c}=\tilde{\theta}_{0}^{c}\left(\theta^{c}, \Gamma^{c}, \Lambda^{c}, \Omega^{c}\right) \\
=-\tan ^{-1}\left[\left(\lambda \Lambda^{c}+\mathcal{F} \tanh \left[t \mathcal{F}-\tanh ^{-1}\left[\frac { 1 } { \mathcal { F } } \left(\lambda \Lambda^{c}+\right.\right.\right.\right.\right. \\
\left.\left.\left.\left.\left.\left(\lambda \Gamma^{c}+\Omega^{c}\right) \tan \theta^{c}\right)\right]\right]\right)\left(\Gamma^{c} \lambda+\Omega^{c}\right)^{-1}\right] .
\end{aligned}
$$

\section{SIMILARITIES TO THE KURAMOTO MODEL}

The orientation evolution equation, Eq. (16), bears a close resemblance to the equation governing a system of coupled oscillators described by the Kuramoto model ${ }^{20}$ in which a collection of $N$ coupled oscillators with phases $\theta_{i}$ interact through a sinusoidal coupling

$$
\dot{\theta}_{i}=\omega_{i}+\frac{K}{N} \sum_{j=1}^{N} \sin \left(\theta_{j}-\theta_{i}\right), \quad i=1, \ldots, N
$$

where $K$ is the coupling strength and the frequencies $\omega_{i}$ follow a symmetric probability density, such as a normal distribution. 

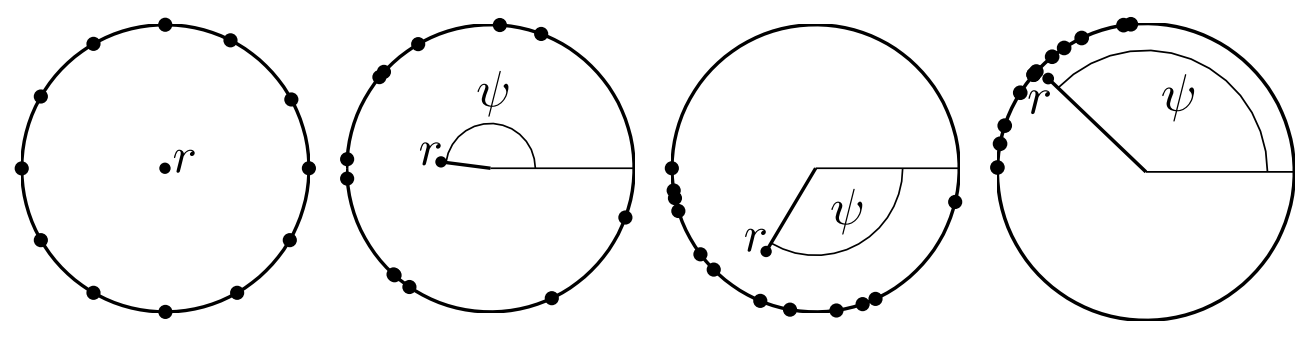

FIG. 2: Geometric representation of the order parameter, with the corresponding $\theta_{j}$ plotted on the unit circle

In order to simplify Eq. (20), as discussed in Strogatz ${ }^{21}$, it is convenient to introduce the complex order parameter

$$
r \mathrm{e}^{i \psi}=\frac{1}{N} \sum_{j=1}^{N} \mathrm{e}^{i \theta_{j}}
$$

where the radius $r$ measures the spread of the $\theta_{j}$ and $\psi$ is the average $\theta_{j}$ (cf. Fig. 2). Kuramoto rewrote Eq. (20) through the order parameter by first, multiplying both sides of Eq. (21) by $\mathrm{e}^{-i \theta_{i}}$ and taking the imaginary part to obtain

$$
r \sin \left(\psi-\theta_{i}\right)=\frac{1}{N} \sum_{j=1}^{N} \sin \left(\theta_{j}-\theta_{i}\right) .
$$

Substituting Eq. (22) into Eq. (20) gives

$$
\dot{\theta}_{i}(t)=\omega_{i}+K r(t) \sin \left(\psi(t)-\theta_{i}(t)\right), \quad i=1, \ldots, N,
$$

As seen above, the phase of each oscillator tends towards the average phase $\psi$ with strength proportional to the radius $r$. Considering steady solutions, where $r(t)$ is constant and $\psi(t)$ rotates uniformly, along with judicious choice of a rotating frame such that $\psi=0$, Eq. (23) reduces to

$$
\dot{\theta}_{i}=\omega_{i}-K r \sin \theta_{i}
$$

which is essentially Eq. 16 with $\theta_{i}=2 \theta^{c}, \omega_{i}=-\Omega^{c}, K r=\lambda \Lambda^{c}$, and $\Gamma^{c}=0$.

The long-term response of an oscillator, i.e. $t \rightarrow \infty$, in this steady treatment of the Kuramoto model leads to two distinct types of behavior, depending on the relationship between the product of the coupling strength and spread $K r$ and the oscillator's natural 
frequency $\omega_{i}$. When $K r \geq\left|\omega_{i}\right|$, the coupling dominates and the oscillator approaches a stable fixed-point at which it is phase-locked, i.e. $\dot{\theta}_{i}=0$; when $K r<\left|\omega_{i}\right|$, locking cannot occur and the oscillator drifts in a nonuniform manner.

In Prantil et al. ${ }^{9}$, the long-term response of the ODF generated using the FCM was found to depend on the relationship between $\lambda$ (cf. Eq. (17)) and

$$
\eta=\tilde{\eta}\left(\Omega^{c}, \Gamma^{c}, \Lambda^{c}\right)=\frac{\left|\Omega^{c}\right|}{\sqrt{\Gamma^{c^{2}}+\Lambda^{c^{2}}}}=\frac{|\Omega|}{\sqrt{\Gamma^{2}+\Lambda^{2}}},
$$

where $\Omega, \Gamma$, and $\Lambda$ are the spin, the rate of shearing, and the rate of stretching, respectively, of the applied velocity gradient $\mathbf{L}$. The parameter $\lambda$, defined by the structure of the undeformed crystal, represents a coupling between the crystals due to their similar crystal structure, whereas $\eta$, defined by $\mathbf{L}$, represents the natural oscillation of the crystal induced by the loading. When $\lambda \geq \eta$, the internal structure dominates, causing the crystals to approach a single orientation $\theta^{p}=\tan ^{-1}(\mathcal{F}-\lambda \Lambda) /(\lambda \Gamma+\Omega)$, i.e. in Eq. (21) $\psi \rightarrow \theta^{p}$ and $r \rightarrow 1$ as shown in Fig. 3(a). When $\lambda<\eta$, the polycrystal experiences "tumbling" behavior where

the texture evolves periodically with a period of $T=\pi / \sqrt{\left(\Gamma^{2}+\Lambda^{2}\right)\left(\eta^{2}-\lambda^{2}\right)}$ as shown in Figs. $3(\mathrm{~d})$ and $3(\mathrm{~g})$. Note that the criteria for the development of a sharp texture $(\lambda \geq \eta)$ and tumbling $(\lambda<\eta)$ correspond respectively to the phase-locking $(K r \geq|\omega|)$ and drifting $(K r<|\omega|)$ response of oscillators noted in the steady treatment of the Kuramoto model.

The Kuramoto model was originally developed as an abstract model to mathematically study the synchronization of a system of coupled oscillators. It was not thought to represent any known system, but rather act as a tool for understanding synchronization in general terms. For this reason, the similarity between the orientation evolution equation of the planar polycrystal and the Kuramoto model is remarkable, albeit that the kinematically determined planar polycrystal is a simplification of "true" polycrystal behavior.

\section{CPFEM RESULTS}

We investigate the texture evolution of the planar polycrystal using the CPFEM. The CPFEM intrinsically accounts for the crystal interactions and therefore provides an accurate representation of the crystal behavior, though it is also computationally expensive. In our analysis, a square polycrystal consisting of 900 crystals with a uniform initial texture distribution is deformed by a constant velocity gradient. It is modeled using 900 square 
elements on a $30 \times 30$ uniform grid, where each element represents one crystal. The representation of uniform crystal shape and size is unrealistic, but does provide a simplified view of the crystal behavior. The CPFEM analyses use the two slip systems described in Eq. (11) with $\beta=\pi / 6$ in which the elastic constants and parameters for the crystal flow rule are taken from the Tantalum parameters presented in Bronkhorst et al. ${ }^{22}$ with no hardening or adiabatic heating. As elastic strains are quite small, the incorporation of elasticity in these CPFEM computations does not significantly affect the texture evolution. The purely 2D planar polycrystal is numerically challenging, and therefore the CPFEM analyses are limited to lower strains than those in mean-field models.

The texture evolution predicted by the CPFEM is significantly different from that predicted by the FCM, as seen by comparing the order parameter trajectories, cf. Eq. (21), predicted by the two methods. When $\lambda \geq \eta$ (Fig. 3(b)), the CPFEM analysis predicts a texture that tends towards a single orientation but the crystals never completely align, i.e. $\psi \rightarrow \theta^{p}$ but $r \rightarrow c$, where $c<1$, rather than $r \rightarrow 1$. When $\lambda<\eta$ (Figs. 3(e) and 3(h)), the CPFEM predicts a decaying texture oscillation. Therefore, the FCM predicts an overly sharp texture when $\lambda \geq \eta$, and a drastically different tumbling texture when $\lambda<\eta$.

The CPFEM analyses also allow us to observe the spatial distribution of the texture evolution in the polycrystal. When $\lambda \geq \eta$ (Figs. 4(a) and 4(b)), the crystals (elements) throughout the mesh evolve towards a single orientation. When $\lambda<\eta$, the mesh contains bands of crystals of similar crystallographic orientation that shear in a common direction, with bands having an alternating sense of shear. In the mesh shown in Figs. 4(c) - 4(f) several of these bands are present, while in the mesh shown in Figs. 4(g)-4(j) the bands are just beginning to form (specific bands of crystals are outlined in black in the figures). It appears that decaying oscillation predicted by the CPFEM may result from transient oscillatory patterns where two groups of crystals, arranged in alternating bands, synchronize in anti-phase.

\section{THE STOCHASTIC TAYLOR MODEL}

The CPFEM results show interesting aspects of the texture evolution. However, due to the complexity of the CPFEM, it is difficult to gain a more comprehensive view. A stochas-

tic mean-field model provides a simple framework to describe the behavior and does not 

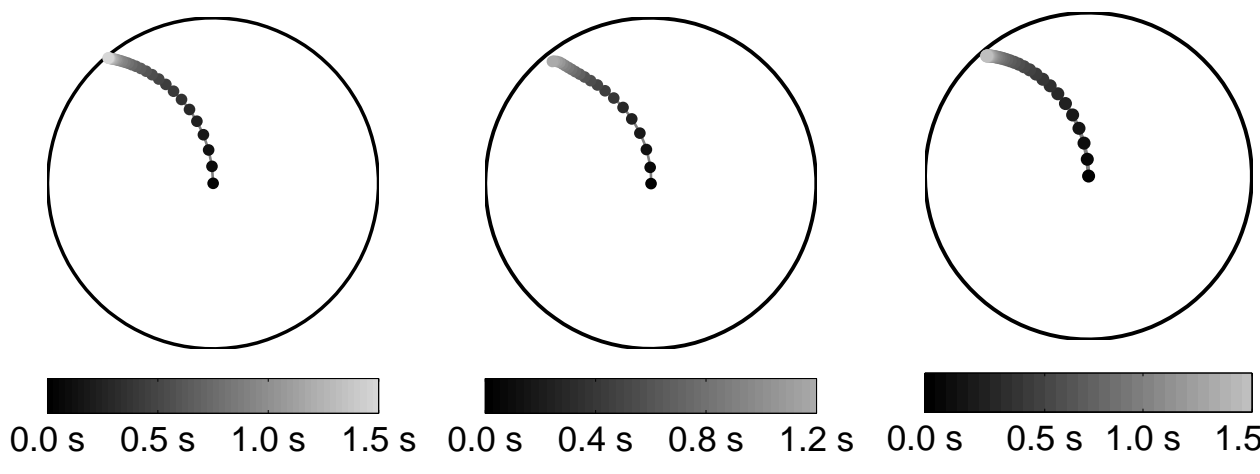

(a)FCM.
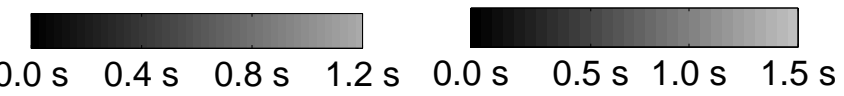

(b)CPFEM.

(c)STM, $\sigma=0.15$.

$\Omega=-1.3, \Gamma=1$, and $\Lambda=0$
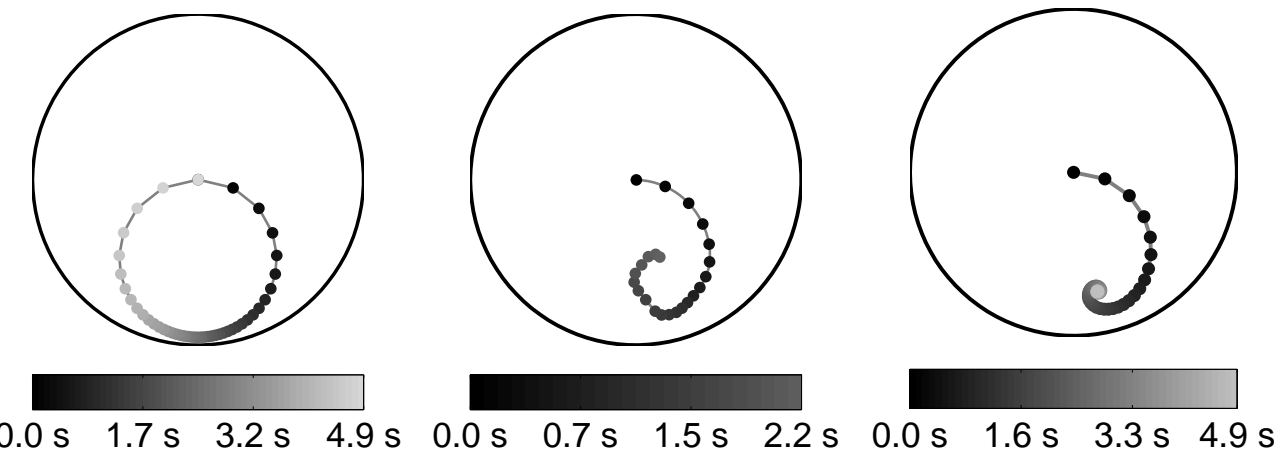

(d)FCM.

(e)CPFEM.

(f)STM, $\sigma=0.15$.

$\Omega=2.1, \Gamma=0$, and $\Lambda=1$
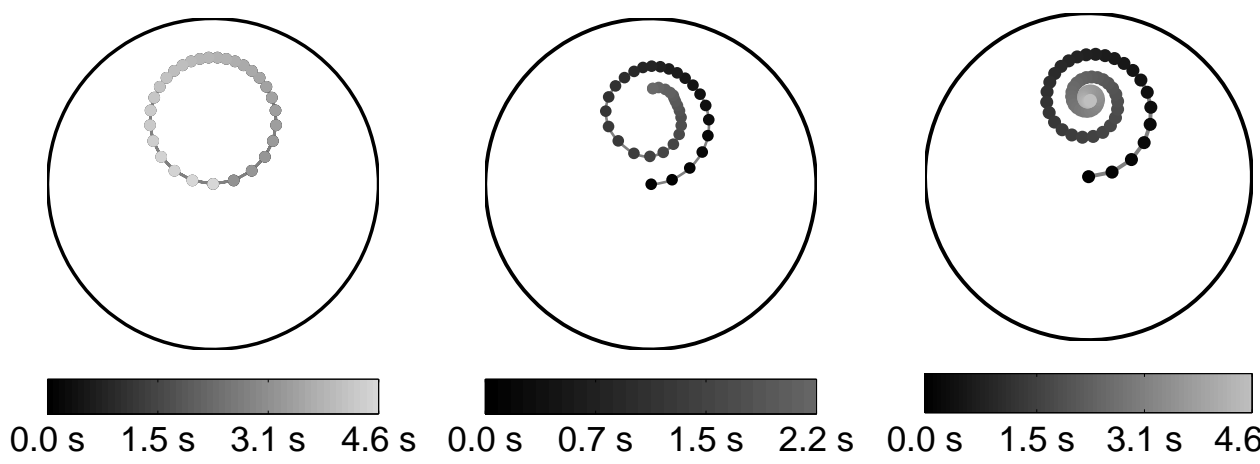

$0.0 s \quad 1.5 s \quad 3.1 s \quad 4.6 s$
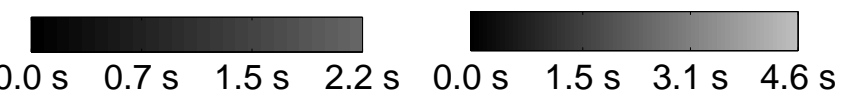

(g)FCM.

(h)CPFEM.

(i)STM, $\sigma=0.12$.

$$
\Omega=-\pi, \Gamma=0 \text {, and } \Lambda=1.2
$$

FIG. 3: The order parameter calculated from Eq. 21 with $\theta_{j}=2 \theta_{c}$ at several time instants for various loadings and simulation methods. The shading of the data points represents the corresponding time as dictated by the scale bar. 

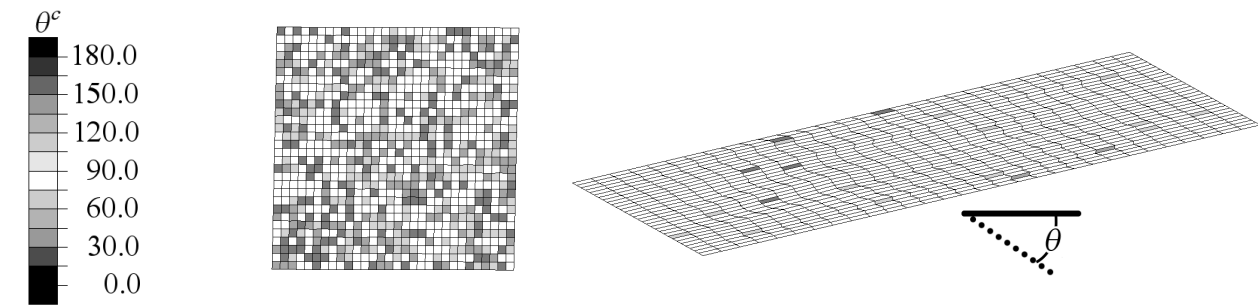

(a) $t=0.0 \mathrm{~s}$.

(b) $t=1.2 \mathrm{~s}$.

$\Omega=-1.3, \Gamma=1$, and $\Lambda=0$

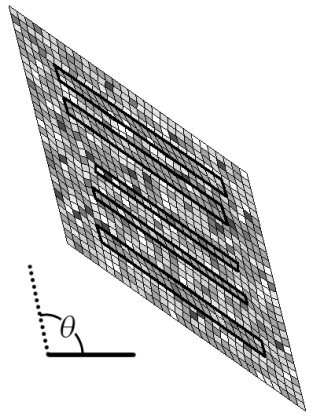

(c) $t=0.71 \mathrm{~s}$.

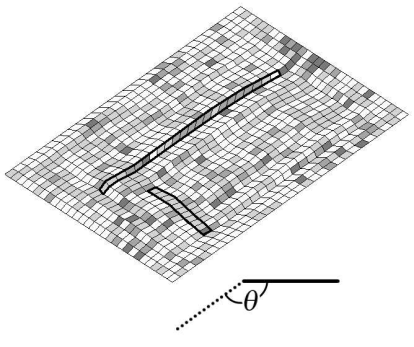

(g) $t=0.85 \mathrm{~s}$.

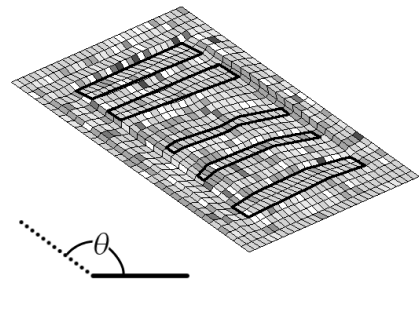

(d) $t=1.29 \mathrm{~s}$. $\Omega=2.1, \Gamma=0$, and $\Lambda=1$
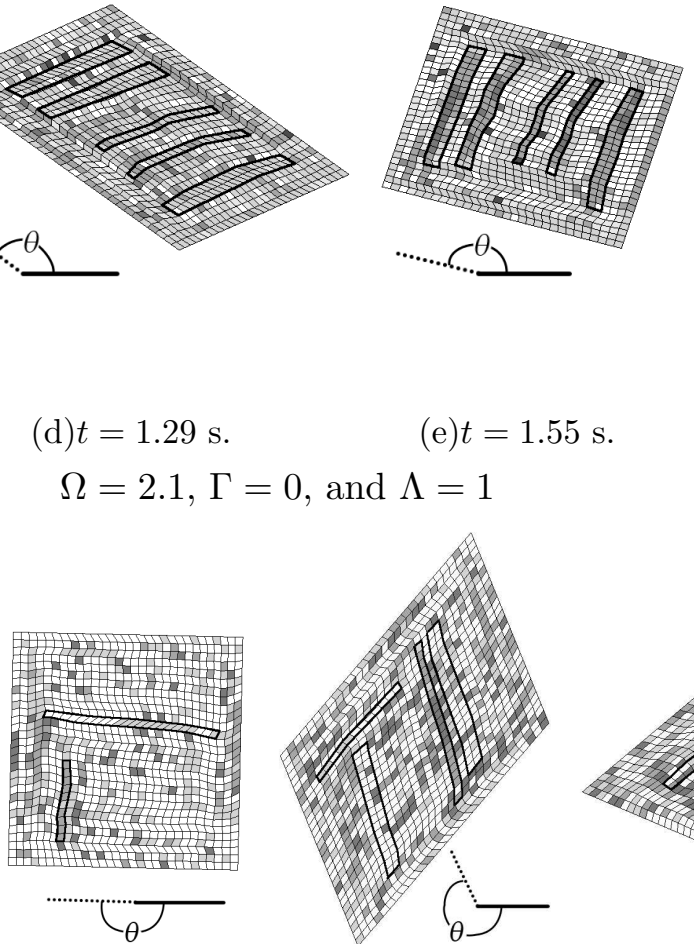

(h) $t=1.09 \mathrm{~s}$.

$\Omega=-\pi, \Gamma=0$, and $\Lambda=1.2$

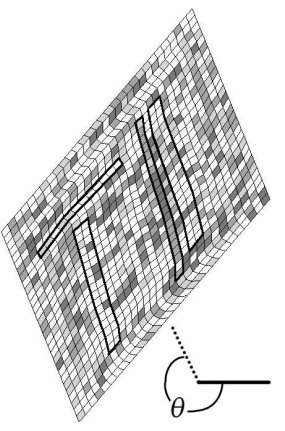

(i) $t=1.38 \mathrm{~s}$. (j) $t=1.87 \mathrm{~s}$.

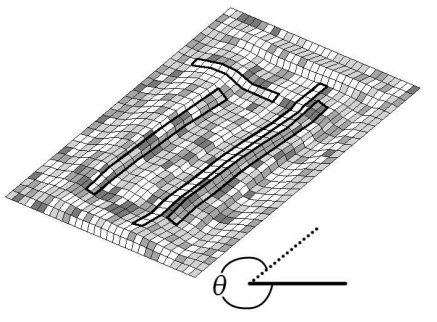

FIG. 4: The deformed mesh at the indicated times due to indicated macro-deformation, where $\theta$ is the rigid-body rotation of the polycrstal at the time $t$. The shading of each element represents the orientation of the crystal in degrees as dictated by the legend. Elements of interest in the mesh are outlined in black.

over-constrain the crystals as does the FCM. Rather than periodic oscillation, the CPFEM predicts decaying oscillation, such that the texture may approach a stable solution. The introduction of random variation into a system can lead to stability ${ }^{23}$, therefore a stochas- 

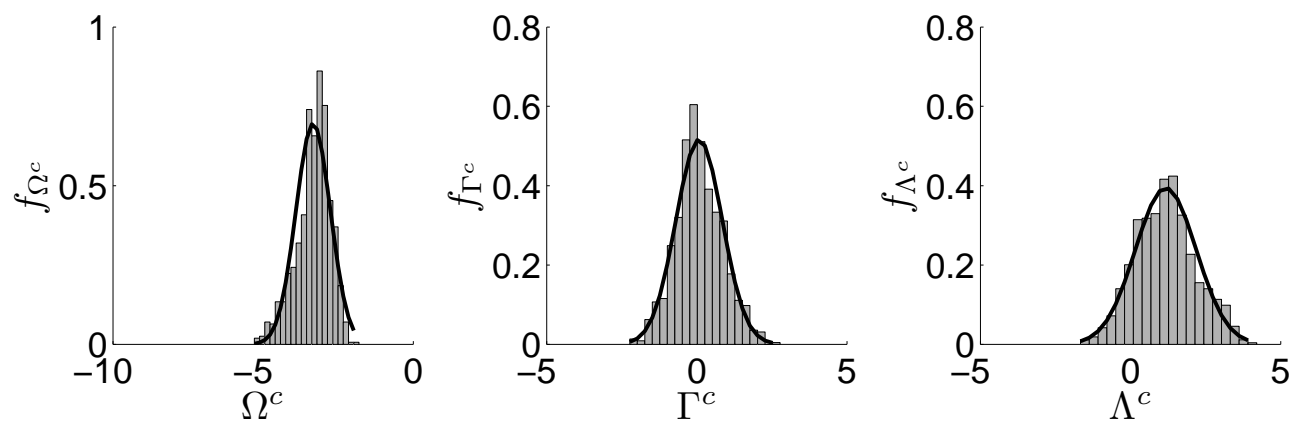

FIG. 5: $f_{\Lambda^{c}}, f_{\Gamma^{c}}$, and $f_{\Omega^{c}}$ from CPFEM with $\Omega=-\pi, \Gamma=0$, and $\Lambda=1.2$ at $t=2.2 \mathrm{~s}$. The bars are a histogram approximation of the PDF and the black line is a normal distribution with the measured $\mu$ and $\sigma$.

tic approach adopted at the meso-scale may result in texture evolution consistent with the CPFEM results.

In Engler ${ }^{24}$ and Ma et al. ${ }^{17}$, CPFEM results drive the application of stochastic variations to obtain $\mathbf{L}^{c}$ from $\mathbf{L}$. Using the CPFEM to model 3D copper polycrystals, Sarma and Dawson ${ }^{25}$ found that the components of the strain rate $\mathbf{D}^{c}$, behave as random variables following a normal distribution with mean value approximately equal to its macro-scale counterpart, i.e. $\mu_{\mathbf{D}^{c}}=\mathbf{D}$. Furthermore, they found that stochastic behavior of the components are uncorrelated and leveraged these results in a viscoplastic model with non-uniform deformation among component crystals $^{26}$. Using this same information, Engler ${ }^{24}$ and Ma et al. ${ }^{17}$ modify mean-field models such that $\mathbf{D}^{c}$ experiences random variations in order to loosen the imposed constraints. Ma et al. ${ }^{17}$ modify the FCM so that $\mathbf{D}^{c}$ follows a normal distribution with mean equal to $\mathbf{D}$ while the skew symmetric part $\mathbf{W}^{c}=\mathbf{W}$. This model, which we call the stochastic Taylor model (STM), gives more accurate predictions than the FCM, however it is based on the results from a single CPFEM analysis.

For the planar polycrystal, our CPFEM results support the assumption that $\mathbf{D}^{c}$ follows a normal distribution with $\mu_{\mathbf{D}^{c}}=\mathbf{D}$ (see Fig. 5 for an example of the probability density functions $f_{\Lambda^{c}}$ and $f_{\Gamma^{c}}$ from CPFEM). In addition, The CPFEM results show that $\mathbf{W}^{c}$ follows a normal distribution (also see Fig. 5 for an example of $f_{\Omega^{c}}$ ). Of the two parameters that define a normal distribution, the mean of $\mathbf{L}^{c}$ is constant but the standard deviation need not be. The CPFEM results show that the component standard deviations change with time, with $\sigma_{\Omega^{c}}$ and $\sigma_{\Gamma^{c}}$ behaving in a somewhat similar manner (cf. Fig. 6). 


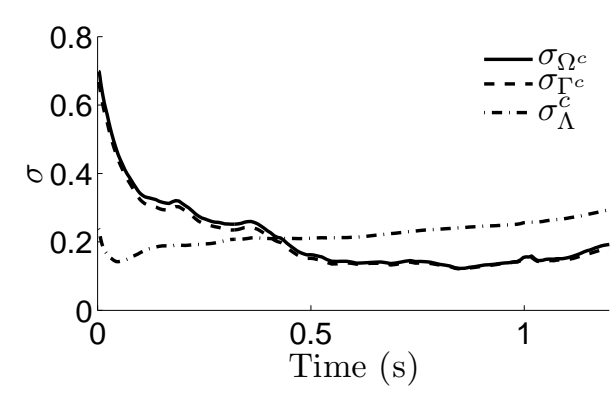

(a) $\Omega=-1.3, \Gamma=1$, and $\Lambda=0$.

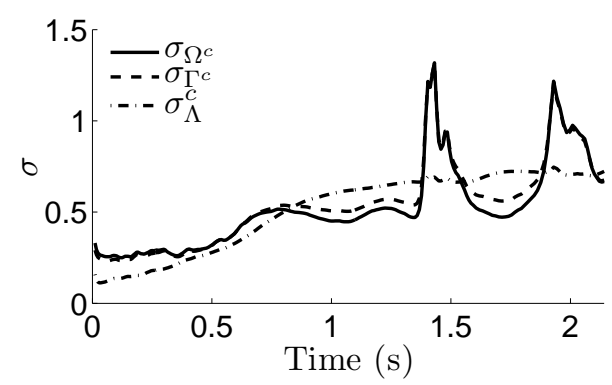

(b) $\Omega=2.1, \Gamma=0$, and $\Lambda=1$.

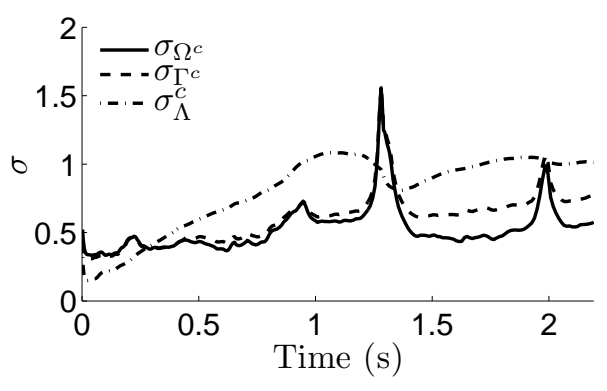

(c) $\Omega=-\pi, \Gamma=0$, and $\Lambda=1.2$.

FIG. 6: The standard deviations of $\Omega^{c}, \Lambda^{c}$, and $\Gamma^{c}$ with time from CPFEM analyses.

The statistical description of the $\mathbf{L}^{c}$ taken from the CPFEM results provides guidance when applying the STM to the planar polycrystal. As discussed above, the CPFEM simulations show that both the skew and symmetric parts of $\mathbf{L}^{c}$ follow a normal distribution, though we treat only the symmetric part as random to provide an objective statistical description. In addition, we simplify the analysis by treating the standard deviations as constant, though the CPFEM results show this assumption to be incorrect. Therefore, assuming no significant correlation, the probability density functions $(\mathrm{PDF})$ for the rate of stretching $f_{\Lambda^{c}}$ and the rate of shearing $f_{\Gamma^{c}}$ are assumed Gaussian, i.e.

$$
\begin{aligned}
& f_{\Gamma^{c}}(x)=\frac{e^{-\frac{(-\Gamma+x)^{2}}{2 \sigma^{2}}}}{\sqrt{2 \pi} \sigma} \\
& f_{\Lambda^{c}}(y)=\frac{e^{-\frac{(-\Lambda+y)^{2}}{2 \sigma^{2}}}}{\sqrt{2 \pi} \sigma}
\end{aligned}
$$

where the standard deviations $\sigma_{\Gamma^{c}}=\sigma_{\Lambda^{c}}=\sigma$ and $\Gamma$ and $\Lambda$ are obtained from $\mathbf{D}$. One limitation of this treatment is that the $\mathbf{L}^{c}$ are independent of the crystal orientations. STM analyses predict textures consistent with that obtained from the CPFEM analyses as shown in Figs. 3(c), 3(f), and 3(i). The STM simulations, which can be performed for longer durations than the CPFEM simulations, result in the order parameter settling in a small 
neighborhood within the unit circle. This signifies a final texture that is either static or oscillatory with constant order parameter.

\section{THE ORIENTATION DISTRIBUTION FUNCTION}

Due to the simple nature of the FCM and the STM, expressions can be developed to describe the texture of the polycrystal at any time $t$. Equation (18) provides a means to find the orientation of an individual crystal, however the texture of the entire polycrystal is characterized by the PDF of the crystal orientation, i.e. $f_{\Theta^{c}}$, the orientation distribution function $(\mathrm{ODF})$. Given the ODF of the undeformed polycrystal, $f_{\Theta_{0}^{c}}\left(\theta_{0}^{c}\right)$, the ODF of the deformed polycrystal is found from ${ }^{27}$

$$
f_{\Theta^{c}}\left(\theta^{c}\right)=f_{\Theta_{0}^{c}}\left(\tilde{\theta}_{0}^{c}\left(\theta^{c}, \Gamma^{c}, \Lambda^{c}, \Omega^{c}\right)\right)\left|\partial_{\theta^{c}} \tilde{\theta}_{0}\left(\theta^{c}, \Gamma^{c}, \Lambda^{c}, \Omega^{c}\right)\right| .
$$

In the above, $f_{\Theta_{0}^{c}}$ and $\mathbf{L}$ (defined by $\Gamma, \Lambda$, and $\Omega$ ) are known, however the $\mathbf{L}^{c}$ (defined by the $\Gamma^{c}, \Lambda^{c}$, and $\Omega^{c}$ ) are unknown.

Using the FCM and a constant deformation, $\Gamma^{c}(t)=\Gamma, \Lambda^{c}(t)=\Lambda$, and $\Omega^{c}(t)=\Omega$ allows us to evaluate $\partial_{\theta^{c}} \tilde{\theta}_{0}^{c}\left(\theta^{c}, \Gamma^{c}, \Lambda^{c}, \Omega^{c}\right)$ from Eq. (19) to obtain

$$
\partial_{\theta^{c}} \tilde{\theta}_{0}^{c}=\frac{\sec \theta^{c^{2}} / \cosh (t \mathcal{F}-\mathcal{W})^{2}}{\left(1-\frac{\left(\lambda \Lambda+(\lambda \Gamma+\Omega) \tan \theta^{c}\right)^{2}}{\mathcal{F}^{2}}\right)\left(1+\frac{(\lambda \Lambda+\mathcal{F} \tanh (t \mathcal{F}-\mathcal{W}))^{2}}{(\lambda \Gamma+\Omega)^{2}}\right)}
$$

where

$$
\mathcal{W}=\tanh ^{-1}\left[\frac{1}{\mathcal{F}}\left(\lambda \Lambda+(\lambda \Gamma+\Omega) \tan \theta^{c}\right)\right] .
$$

For an uniform initial texture distribution i.e., there is an equal chance of getting any orientation giving $f_{\Theta_{0}^{c}}\left(\theta_{0}^{c}\right)=1 / \pi$, the ODF becomes

$$
f_{\Theta^{c}}\left(\theta^{c}\right)=\frac{\sec \theta^{c} / \cosh (t \mathcal{F}-\mathcal{W})^{2}}{\pi\left(1-\frac{\left(\lambda \Lambda+(\lambda \Gamma+\Omega) \tan \theta^{c}\right)^{2}}{\mathcal{F}^{2}}\right)\left(1+\frac{(\lambda \Lambda+\mathcal{F} \tanh (t \mathcal{F}-\mathcal{W}))^{2}}{(\lambda \Gamma+\Omega)^{2}}\right)}
$$

Using the STM (again, for constant values of $\Gamma, \Lambda$, and $\Omega$ and a uniform initial texture) Eq. (18) is a function of three random variables, $\Gamma^{c}, \Lambda^{c}$, and $\theta_{0}^{c}$, with known PDFs (c.f. Eq. 26) whose ODF is obtained from

$$
\begin{gathered}
f_{\Theta^{c}}\left(\theta^{c}\right)=\int_{-\infty}^{\infty} \int_{-\infty}^{\infty} f_{\Theta_{0}^{c}}\left(\tilde{\theta}_{0}^{c}\left(\theta^{c}, x, y, \Omega^{c}\right)\right) f_{\Gamma^{c}}(x) f_{\Lambda^{c}}(y) \\
\\
\qquad \partial_{\theta^{c}} \tilde{\theta}_{0}{ }_{0}\left(\theta^{c}, x, y, \Omega^{c}\right) \mid d x d y .
\end{gathered}
$$


This integral is evaluated numerically, where the limits $\pm \infty$ are replaced with $\pm 10 \sigma$ with $\sigma=\sigma_{\Lambda^{c}}, \sigma_{\Gamma^{c}}$, respectively.

\section{STM ANALYSIS AND DISCUSSION}

The STM predicts texture evolution that more closely resembles that obtained from the CPFEM than that predicted by the FCM. As with the FCM, the behavior predicted by the STM resembles that given by the Kuramoto model. However, now the steady and oscillatory behavior is determined by the relationship between $\lambda$ and $\eta^{c}=\left|\Omega^{c}\right| / \sqrt{\Gamma^{c 2}+\Lambda^{c 2}}$. A function of random variables, $\eta^{c}$ has a PDF

$$
f_{\eta^{c}}\left(\eta^{c}\right)=\int_{-\infty}^{\infty} f_{\Gamma^{c}}(\overbrace{\sqrt{\Omega^{c^{2}} / \eta^{c}-x^{2}}}^{\Gamma^{c}}) f_{\Lambda^{c}}(x)\left|\partial_{\eta^{c}} \tilde{\Gamma}^{c}\left(\eta^{c}, x, \Omega^{c}\right)\right| d x
$$

where, following the derivation of Eq. (31), we "invert" Eq. (25) to obtain an expression for $\Gamma^{c}$. The relationship between $\lambda$ and $\eta^{c}$ varies in each crystal, such that the behavior of the polycrystal depends on the probability that $\lambda>\eta^{c}$, i.e. $P\left(\lambda>\eta^{c}\right)$. When $P\left(\lambda>\eta^{c}\right)$ is high, the crystal structure dominates and the texture evolves towards a single orientation (Fig. $3(\mathrm{c}))$. On the other hand, when $P\left(\lambda>\eta^{c}\right)$ is low, the load dominates and a tumbling-like behavior is exhibited (Fig. 3(i)). In all other cases, an intermediate behavior is observed (Fig. 3(f)).

Different values of $\sigma$ lead to different $\operatorname{PDF} f_{\eta^{c}}$ behavior and thus different values of $P\left(\lambda>\eta^{c}\right)$, as seen in Fig. 7. With increasing values of $\sigma$, the standard deviation of $\eta^{c}$, $\sigma_{\eta^{c}}$, increases and hence, more intermediate behavior is observed. Consequently, the same L produces significantly different ODF histories depending on the standard deviations.

An interesting aspect of the texture evolution behavior exhibited by the STM and CPFEM is that the texture evolves towards a steady ODF for any load. To understand this apparently steady texture distribution, we use the STM to model the behavior of the order parameter $r$ over time and the shape of the steady-state ODF. The time it takes for $r$ to reach a constant value (Fig. 8) depends on the applied load L, through $\eta$, and on the standard deviations $\sigma=\sigma_{\Gamma^{c}}=\sigma_{\Lambda^{c}}$, i.e. increasing $\eta / \lambda$ increases the time while increasing $\sigma$ decreases the time. The shape of the steady-state ODF (Fig. 9) also depends on $\eta$ and $\sigma$. As $\sigma$ and $\eta / \lambda$ increase, the standard deviation of the orientation $\sigma_{\Theta^{c}}$ increases, though the sensitivity of $\sigma_{\Theta^{c}}$ to $\sigma$ decreases with increasing $\eta / \lambda$.

JAM-07-1070, Tonks, 16 

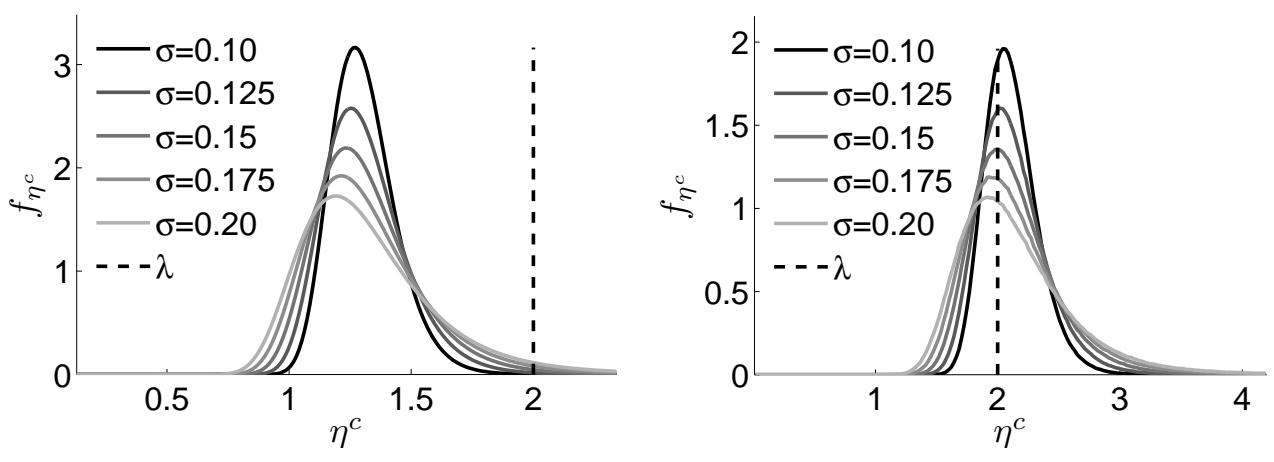

(a) $\Omega=-1.3, \Gamma=1$, and $\Lambda=0$.

(b) $\Omega=2.1, \Gamma=0$, and $\Lambda=1$.

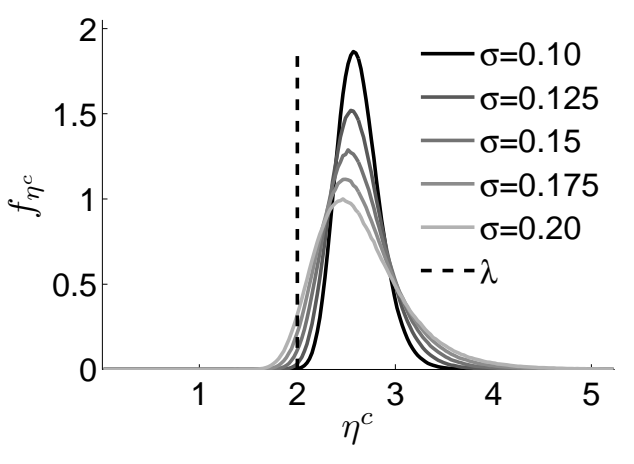

(c) $\Omega=-\pi, \Gamma=0$, and $\Lambda=1.2$.

FIG. 7: Relationship between the PDF of $\eta^{c}$ and $\lambda$ for several standard deviations $\sigma=\sigma_{\Gamma^{c}}=\sigma_{\Lambda^{c}}$.

The CPFEM and STM predictions of the texture evolution in planar polycrystals with two slip systems are similar but distinct from the findings of Prantil et al. ${ }^{9}$. Whether the texture initially begins to oscillate or evolve towards preferred orientations, the ODF will eventually reach a steady-state value. The time required to reach the steady-state ODF depends on the magnitude of the imposed shear and the structure of the crystal slip systems. Once the ODF stops evolving, the crystals may still reorient in a manner such that the overall distribution does not change. Due to the similarities in the findings of Prantil et al. ${ }^{9}$ to those of Kumar and Dawson ${ }^{10}$ and to Kumar ${ }^{11}$, we assume similar behavior would occur in planar polcyrystals with more than two slip systems and 3D polycrystals, though further research is warranted.

The STM proved to be a valuable tool in analyzing the texture evolution of the planar polycrystal. By introducing stochastic variation into the crystal strain rates, behavior similar to that shown by the CPFEM was predicted, yet the model was simple enough to allow explicit expressions to be developed to describe the texture behavior. The STM could prove 

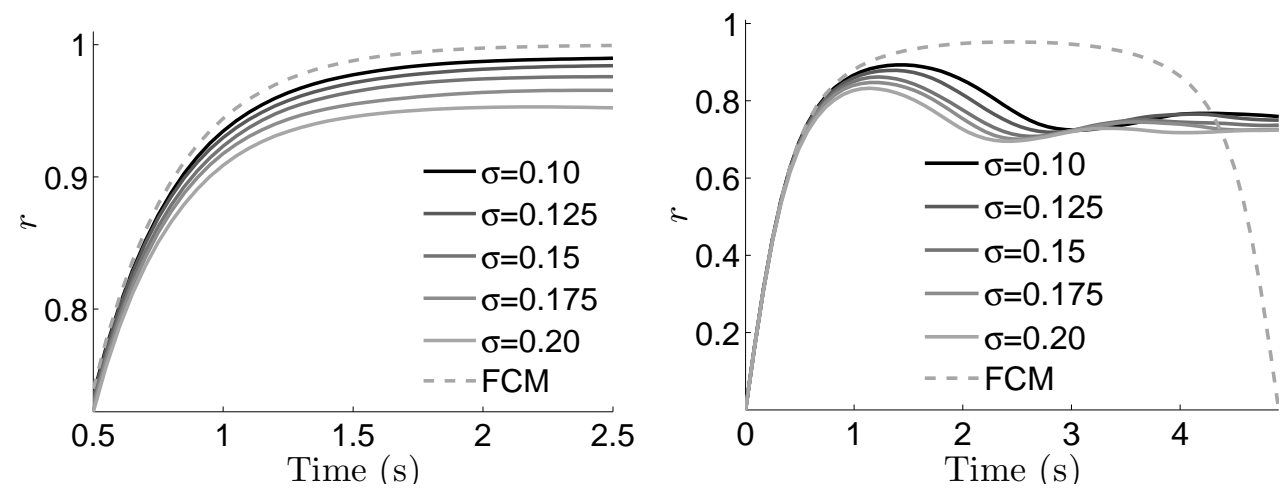

$$
\text { (a) } \Omega=-1.3, \Gamma=1 \text {, and } \Lambda=0 . \quad \text { (b) } \Omega=2.1, \Gamma=0, \text { and } \Lambda=1 .
$$

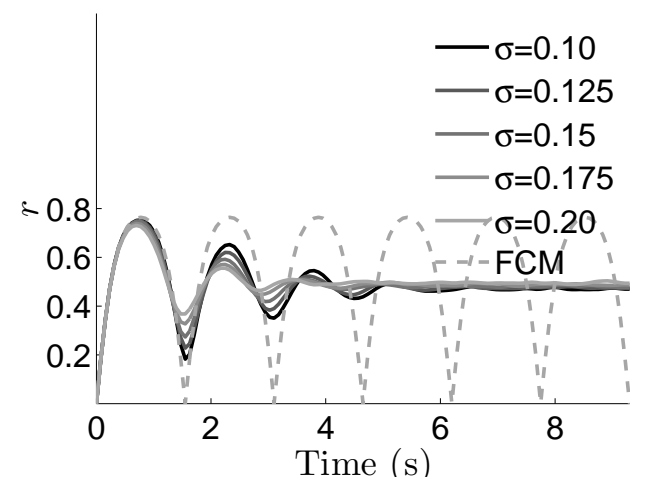

$$
\text { (c) } \Omega=-\pi, \Gamma=0 \text {, and } \Lambda=1.2 \text {. }
$$

FIG. 8: $r$ vs. time at several standard deviations $\sigma=\sigma_{\Gamma^{c}}=\sigma_{\Lambda^{c}}$.

to be a valuable method for mean-field polycrystal plasticity, though care must be taken to identify representative standard deviations.

\section{CONCLUSIONS}

The planar polycrystal plasticity treatment from Prantil et al. ${ }^{9}$ establishes a simplified framework for observing polycrystal texture evolution, revealing that the planar texture evolution equation is similar to the Kuramoto model of a system of coupled oscillators. By simulating the texture evolution of the planar polycrystal using the CPFEM and the STM, we find that the polycrystals reach a steady-state ODF not predicted by the FCM. In the CPFEM, the steady-state ODF seems to be due to the formation of alternating bands of crystals with orientations synchronized in anti-phase. The question as to whether similar behavior would result from applying the CPFEM to planar polycrystals with more slip systems and to 3D polycrystals is a topic that warrants further study. Additionally, the 

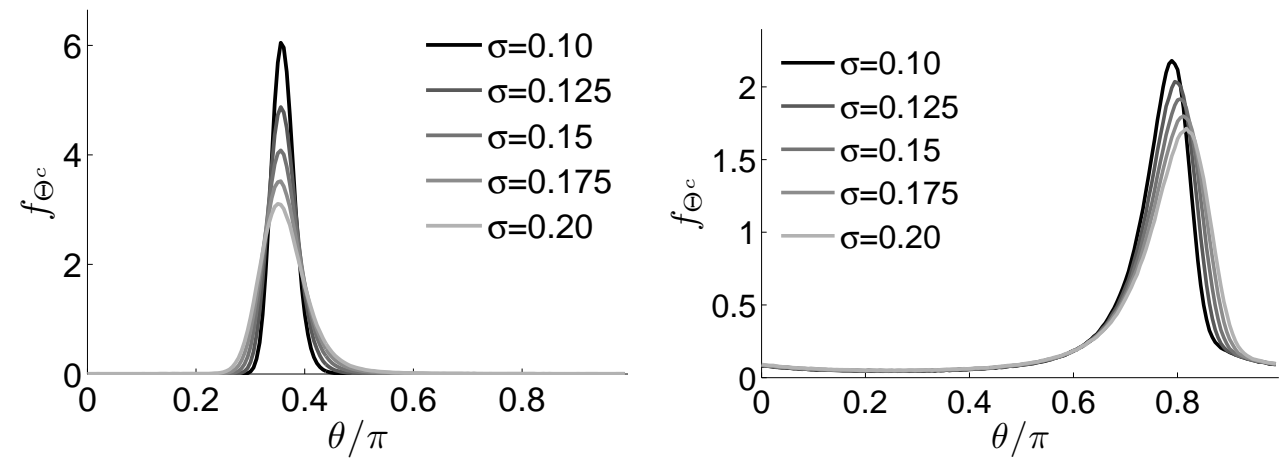

$$
\text { (a) } \Omega=-1.3, \Gamma=1 \text {, and } \Lambda=0 . \quad \text { (b) } \Omega=2.1, \Gamma=0, \text { and } \Lambda=1 .
$$

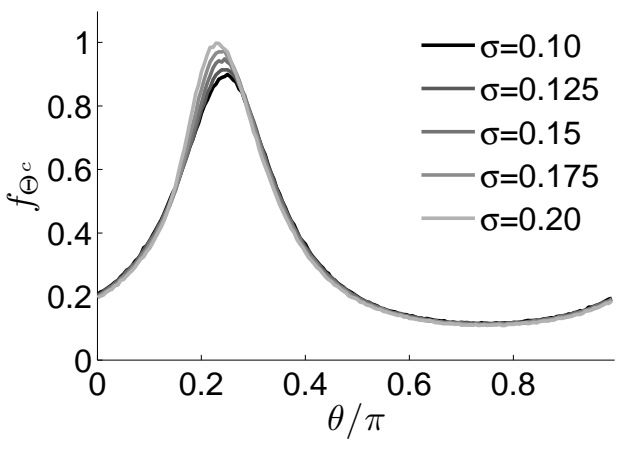

$$
\text { (c) } \Omega=-\pi, \Gamma=0 \text {, and } \Lambda=1.2 \text {. }
$$

FIG. 9: Steady-state ODF at several standard deviations $\sigma=\sigma_{\Gamma^{c}}=\sigma_{\Lambda^{c}}$.

STM appears to be a useful homogenization method, resulting in increased accuracy over the FCM when using representative standard deviations.

1 P. Van Houtte and A. Van Bael. Multi-level versus heirarchical modelling of the plastic deformation of polycrystalline materials implementation in fe codes. In Proceedings of the International Symposium on Plasticity, 2005.

2 G.I. Taylor. Plastic strain in metals. J. Inst. Metals, 62:307 - 324, 1938.

3 S. V. Harren and R. J. Asaro. Nonuniform deformations in polycrystals and aspects of the validity of the taylor model. J. of Mech. Phys. Solids, 37(2):191 - 232, 1989.

4 S.R. Kalidindi, C.A. Bronkhorst, and L. Anand. Crystallographic texture evolution during bulk deformation processing of fcc metals. J. of Mech. Phys. Solids, 40:537 - 569, 1992.

5 H. Honneff and H. Mecking. Analysis of the deformation texture at different rolling conditions. Proceedings of ICOTOM 6, 1:347 - 355, 1982. 
6 U.F. Kocks and H. Chandra. Slip geometry in partially constrained deformation. Acta Metall., 30:695, 1982.

7 P. Van Houtte. On the equivalence of the relaxed taylor theory and the bishophill theory for partially constrained plastic deformation of crystals. Mater. Sci. Eng., 55:69 - 77, 1982.

8 R.A. Lebensohn and C N Tomé. A self-consistent anisotropic approach for the simulation of plastic deformation and texture development of polycrystals: application to zirconium alloys. Acta Metall. Mater., 41:2611 - 2624, 1993.

9 V. C. Prantil, J. T. Jenkins, and P. R. Dawson. An analysis of texture and plastic spin for planar polycrystals. J. of Mech. Phys. Solids, 41(8):1357 - 1382, 1993.

10 A. Kumar and P. R. Dawson. The simulation of texture evolution with finite elmeents over orientation space ii. application to planar crystals. Comput. methods appl. mech. and Engrg., 130:247-261, 1996.

11 A. Kumar. Polycrystal modeling with finite elements over orientatation space. Ph.D. thesis, Cornell University, 1996.

12 R.J. Asaro and A. Needleman. Texture development and strain hardening in rate dependent polycrystals. Acta Metall, 33:923 - 953, 1985.

13 K.K. Mathur and P.R. Dawson. On modeling the development of crystallographic texture in bulk forming processes. Int. J. of Plasticity, 5(1):67 - 94, 1989.

14 R. Becker. Analysis of texture evolution in channel die compression - i. effects of grain interaction. Acta metallurgica et materialia, 39(6):1211 - 30, 1991.

15 S. R. Kalidindi, C. A. Bronkhorst, and L. Anand. On the accuracy of the taylor assumption in polycrystalline plasticity. In Anisotropy and localization of plastic deformation, pages 139 142. Elsevier appl sci publ ltd, 1991.

16 A. J. Beaudoin, K. K. Mathur, P. R. Dawson, and G. C. Johnson. Three-dimensional deformation process simulation with explicit use of polycrystal plasticity models. Int. J. of Plasticity, 9(7):833-60, 1993.

17 A. Ma, F. Roters, and D. Raabe. Numerical study of textures and lankford values for fcc polycrystals by use of a modified taylor model. Comput. Mater. Sci., 29:353 - 361, 2004.

18 S. R. Arwade and M. Grigoriu. Evolution of crystallographic orientations in crystals subject to random and deterministic deformation. Probabilistic Engineering Mechanics, 18:289 - 299, 2003. 
19 S. Kok, A.J. Beaudoin, and D. A. Tortorelli. A polycrystal plasticity model based on the mechanical threshold. Int. J. of Plasticity, 18:715 - 741, 2002.

20 Y. Kuramoto. Chemical Oscillations, Waves, and Turbulence. Springer, Berlin, 1984.

21 S. H. Strogatz. From kuramoto to crawford: exploring the onset of synchronization in populations of coupled oscillators. Physica D. Nonlinear phenomena, 143(1-4):1 - 20, 2000.

22 C.A. Bronkhorst, B.L. Hansen, E.K. Cerreta, and J.F. Bingert. Modeling the microstructural evolution of metallic polycrystalline materials under localization conditions. J. of Mech. Phys. Solids, 55(11):2351-2383, 2007.

23 Michael C. Mackey, Andre Longtin, and Andrzej Lasota. Noise-induced global asymptotic stability. J. of Statistical Physics, 60(5/6):735 - 751, 1990.

24 O. Engler. A new approach to more realistic rolling texture simulation. Advanced Engineering Materials, 4(4):181 - 186, 2002.

25 G. B. Sarma and P. R. Dawson. Effects of interactions among crystals on the inhomogeneous deformations of polycrystals. Acta Materiala, 44(5):1937 - 1953, 1996.

26 G. B. Sarma and P. R. Dawson. Texture predictions using a polycrystal plasticity model incorporating neighbor interactions. Int. J. of plasticity, 12(8):1023 - 1054, 1996.

27 S. S. Rao. Reliability-based design, chapter 5, pages 115-138. McGraw-Hill, Inc., New York, 1992.

\section{List of Figures}

1 Single planar crystal structure. . . . . . . . . . . . . . . . . . 5

2 Geometric representation of the order parameter, with the corresponding $\theta_{j}$ plotted on the unit circle . . . . . . . . . . . . . . . . 8

3 The order parameter calculated from Eq. 21 with $\theta_{j}=2 \theta_{c}$ at several time instants for various loadings and simulation methods. The shading of the data points represents the corresponding time as dictated by the scale bar. . 11

JAM-07-1070, Tonks, 21 
4 The deformed mesh at the indicated times due to indicated macrodeformation, where $\theta$ is the rigid-body rotation of the polycrstal at the time $t$. The shading of each element represents the orientation of the crystal in degrees as dictated by the legend. Elements of interest in the mesh are outlined in black. . . . . . . . . . . . . . . . . . . . . . 12

$5 f_{\Lambda^{c}}, f_{\Gamma^{c}}$, and $f_{\Omega^{c}}$ from CPFEM with $\Omega=-\pi, \Gamma=0$, and $\Lambda=1.2$ at $t=2.2$ s. The bars are a histogram approximation of the PDF and the black line is a normal distribution with the measured $\mu$ and $\sigma \ldots \ldots \ldots \ldots$

6 The standard deviations of $\Omega^{c}, \Lambda^{c}$, and $\Gamma^{c}$ with time from CPFEM analyses.

7 Relationship between the PDF of $\eta^{c}$ and $\lambda$ for several standard deviations

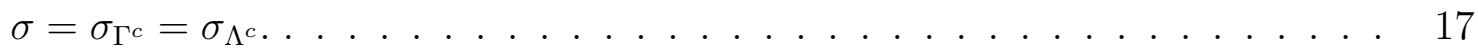

$8 \quad r$ vs. time at several standard deviations $\sigma=\sigma_{\Gamma^{c}}=\sigma_{\Lambda^{c}} \ldots \ldots \ldots$. . . . . . 18

$9 \quad$ Steady-state ODF at several standard deviations $\sigma=\sigma_{\Gamma^{c}}=\sigma_{\Lambda^{c}} . \ldots$. . . . 19 